\title{
GRB 080407: an ultra-long burst discovered by the IPN
}

\section{Pal'shin*}

Ioffe Physical Technical Institute, St. Petersburg, 194021, Russian Federation

E-mail: valomail ioffe.ru

\section{K. Hurley}

U.C. Berkeley Space Sciences Laboratory, 7 Gauss Way, Berkeley, CA 94720-7450, U.S.A.

E-mail: khurleydss1.berkeley.edu

\section{J. Goldsten}

Applied Physics Laboratory, Johns Hopkins University, Laurel, MD 20723, U.S.A.

E-mail: iohn.goldstend jhuapl.edu

\section{G. Mitrofanov}

Institute for Space Research, Profsojuznaja 84/32, Moscow 117997, Russian Federation

E-mail: imitrofaespace.ru

\section{W. Boynton}

Univeristy of Arizona, Lunar and Planetary Laboratory, Tucson, AZ 85721, U.S.A.

E-mail: wboyntondpl.arizona.edu

\section{A. von Kienlin}

Max-Planck-Institut für extraterrestrische Physik, Giessenbachstrasse, Garching, 85748

Germany

E-mail: azkdmpe.mpq.de

\section{J. Cummings}

NASA Goddard Space Flight Center, Code 661, Greenbelt, MD 20771, U.S.A.

E-mail: jaycemilkyway.gsfc.nasa.gov

\section{Feroci}

INAF/IASF-Roma, via Fosso del Cavaliere 100, 00133, Roma, Italy

E-mail: Marce.Ferecidiasf-roma.inaf.it

\section{R. Aptekar, D. Frederiks, S. Golenetskii, E. Mazets, D. Svinkin}

Ioffe Physical Technical Institute, St. Petersburg, 194021, Russian Federation

E-mail: aptekaremail.ioffe.ru, fredamail.ioffe.ru,

golendmail.ioffe.ru, mazetsdmail.ioffe.rul svinkindmail.ioffe.ru

\section{Golovin, M. L. Litvak, A. B. Sanin}

Institute for Space Research, Profsojuznaja 84/32, Moscow 117997, Russian Federation

E-mail: dimamsudmail.ru, maxdcarsmx.iki.rssi.ru, sanindmx.iki.rssi.ru

\section{Fellows, K. Harshman}




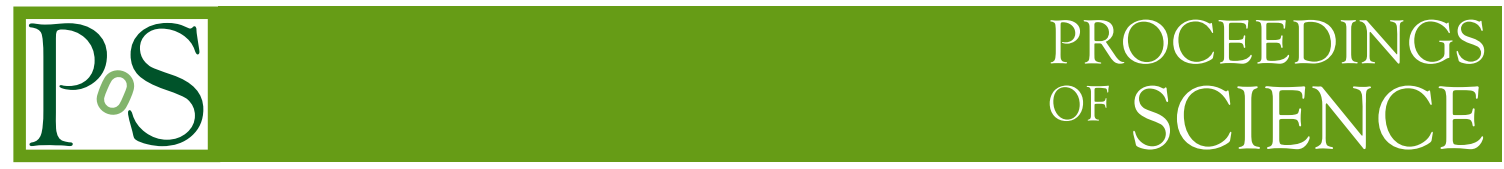

Univeristy of Arizona, Lunar and Planetary Laboratory, Tucson, AZ 85721, U.S.A.

E-mail: Cfellowselpl.arizona.edu, karlelpl.arizona.edu

\section{R. Starr}

Physics Department, The Catholic University of America, Washington, DC 20064, USA

E-mail: richard.starregsfc.nasa.gov

\section{A. Rau, X. Zhang}

Max-Planck-Institut für extraterrestrische Physik, Giessenbachstrasse, Garching, 85748

Germany

E-mail: araudmpe.mpg.de, zhangxdmpe.mpg.de

\section{Savchenko}

ISDC Data Centre for Astrophysics, Ch. d'Ecogia 16, 1290, Versoix, Switzerland

E-mail: Volodymyr.Savchenkodunige.ch

\section{S. D. Barthelmy, N. Gehrels, H. Krimm, D. Palmer}

NASA Goddard Space Flight Center, Code 661, Greenbelt, MD 20771, U.S.A.

E-mail: scottemilkyway.gsfc.nasa.gov gehrelsdheavx.gstc.nasa.gov,

krimmemilkyway.gsfc.nasa.gov, palmerdlanl.gov

\section{E. Del Monte, M. Marisaldi}

INAF/IASF-Roma, via Fosso del Cavaliere 100, 00133, Roma, Italy

E-mail: delmontediasf-roma.inaf.it, marisaldidiasfbo.inaf.it

We present observations of the extremely long GRB 080704 obtained with the instruments of the Interplanetary Network (IPN). The observations reveal two distinct emission episodes, separated by a $\sim 1500 \mathrm{~s}$ long period of quiescence. The total burst duration is about $2100 \mathrm{~s}$. We compare the temporal and spectral characteristics of this burst with those obtained for other ultra-long GRBs and discuss these characteristics in the context of different models.

Gamma-Ray Bursts 2012 Conference-GRB2012,

May 07-11, 2012

Munich, Germany

\footnotetext{
* Speaker.
} 


\section{Observations}

The first emission episode of GRB 080704 was detected at about 74529 s UT (20:42:09) on July 4th 2008. It displays a bright initial pulse followed by two weaker pulses - see Fig. $\mathrm{W}$. The total duration of the episode is $\sim 160 \mathrm{~s}\left(\mathrm{~T}_{90}=142.2 \pm 1.5 \mathrm{~s}\right.$ in the $21-1360 \mathrm{keV}$ band $\left.^{1}\right)$. The whole episode was observed by Konus-Wind, INTEGRAL-SPI-ACS, and Swift-BAT (outside of the coded FoV). The initial pulse was also detected by AGILE (SuperAGILE \& MCAL), Mars Odyssey (GRS \& HEND), and MESSENGER (GRNS).

The second episode was detected at about $76000 \mathrm{~s}$ UT (21:06:40), i.e. $\sim 1500 \mathrm{~s}$ after the onset of the burst. It shows two broad pulses with a total duration of $\sim 400 \mathrm{~s}$ and a weaker emission before the pulses - see Fig. W. Both pulses were detected by INTEGRAL-SPI-ACS, Swift-BAT (outside of the coded FoV), and MESSENGER (GRNS). The first pulse is also detected in the HEND data, however, the time interval of the second pulse is strongly affected by noise spikes. Unfortunately this episode was seen by Konus-Wind only in the housekeeping data ( $80-360 \mathrm{keV}$ range, $3.68 \mathrm{~s}$ resolution) due to data readout after the trigger on the initial pulse, so no spectral data are available for this episode.
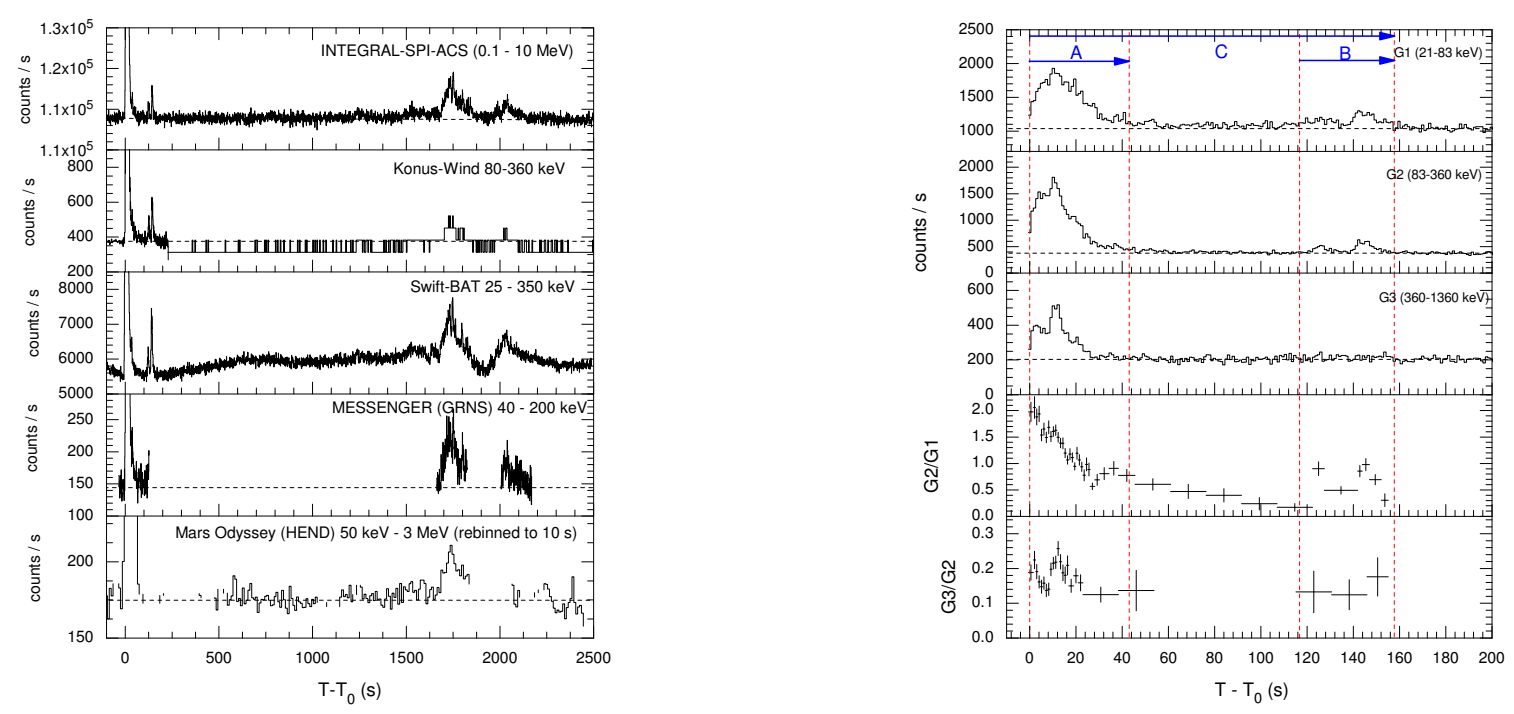

Figure 1: GRB 080407 light curve. Left: full light curve as observed by different IPN instruments. $\mathrm{T}_{0}=$ $\mathrm{T}_{0}^{*}(\mathrm{KW})=74529.471 \mathrm{~s}$ UT $(20: 42: 09.471){ }^{*}-$ corrected for the propagation time delay to Earth; $\mathrm{T}_{0}(\mathrm{KW})$ - the Konus-Wind trigger time). Numerous noise spikes have been removed from the HEND lc. Right: first emission episode in three energy bands and hardness ratios as observed by Konus-Wind.

\section{IPN localization}

We have triangulated the first episode to the rather small 3 sigma error box shown in Fig. $\square$ (left panel). The error box area is $582 \mathrm{sq}$. arcmin, and its maximum dimension is $2.67 \mathrm{deg}$. For the second episode we have derived the rather large 3 sigma error box shown in Fig. $\square$ (right panel). The error box area is $17.1 \mathrm{sq}$. deg. This box contains the small box for the first episode. The coordinates of the boxes are given in Table $\mathbb{C}$.

\footnotetext{
1 as observed by Konus-Wind
} 

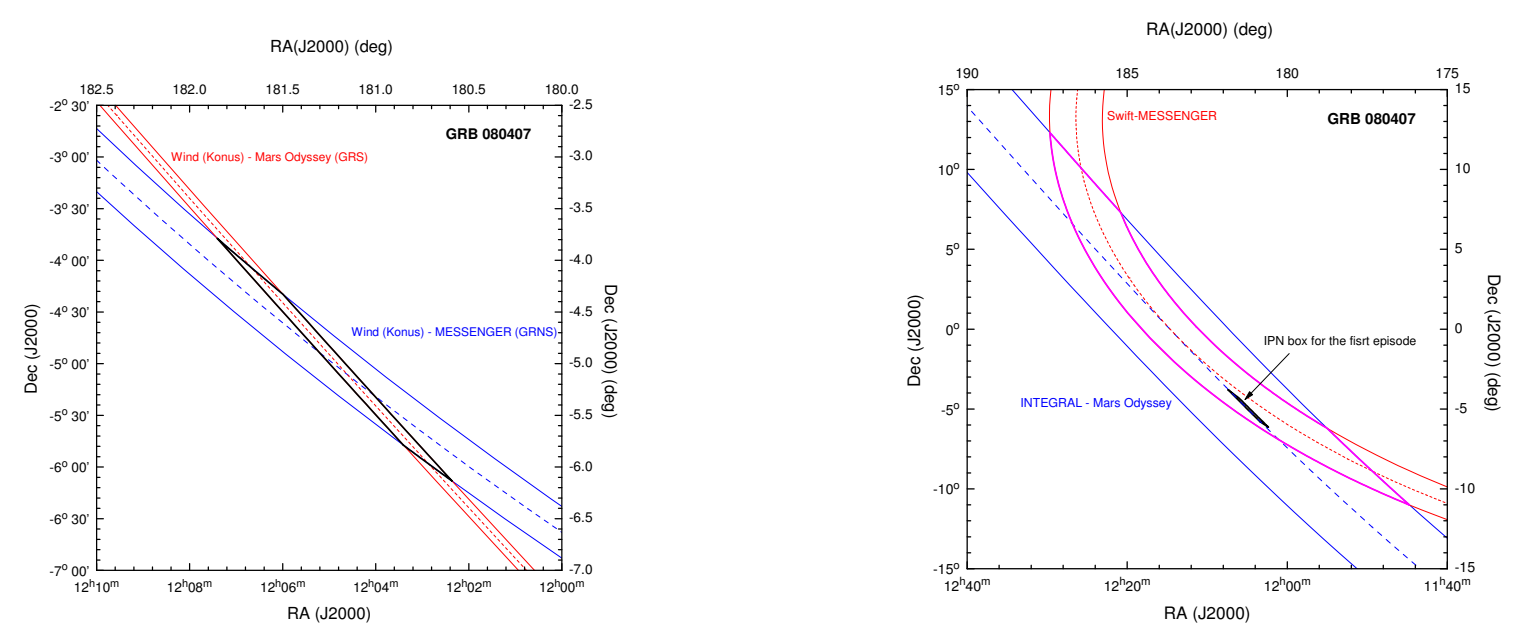

Figure 2: IPN error boxes for GRB 080407. Left: for the first episode. Right: for the second episode.

Table 1: IPN localization of GRB 080407

\begin{tabular}{|c|c|c|c|c|}
\hline & \multicolumn{2}{|c|}{ first episode } & \multicolumn{2}{|c|}{ second episode } \\
\hline & RA(2000), deg & $\operatorname{Dec}(2000)$, deg & RA(2000), deg & Dec(2000), deg \\
\hline Center & 181.151 (12h 04m 36s) & $-5.110\left(-5 \mathrm{~d} 06^{\prime} 34^{\prime \prime}\right)$ & & \\
\hline Corner1 & $180.589(12 \mathrm{~h} 02 \mathrm{~m} 21 \mathrm{~s})$ & $-6.136\left(-6 d 08{ }^{\prime} 11^{\prime \prime}\right)$ & $187.413(12 \mathrm{~h} 29 \mathrm{~m} \mathrm{39s})$ & +12.295 (+12d 17' 42") \\
\hline Corner2 & $180.851(12 \mathrm{~h} 03 \mathrm{~m} \mathrm{24s})$ & $-5.789\left(-5 d 47^{\prime} 22 "\right)$ & $185.204(12 \mathrm{~h} 20 \mathrm{~m} 49 \mathrm{~s})$ & +7.311 (+7d 18'39") \\
\hline Corner3 & $181.852(12 \mathrm{~h} 07 \mathrm{~m} 24 \mathrm{~s})$ & $-3.787(-3 d 47 ’ 13 ")$ & $178.760(11 \mathrm{~h} 55 \mathrm{~m} 02 \mathrm{~s})$ & -6.199 ( -6d 11' $56 ")$ \\
\hline Corner4 & $181.499(12 \mathrm{~h} \mathrm{06m} \mathrm{00s)}$ & $-4.327\left(-4 d 1^{\prime} 38^{\prime \prime}\right)$ & $176.185(11 \mathrm{~h} 44 \mathrm{~m} 44 \mathrm{~s})$ & $-11.005\left(-11 \mathrm{~d} 00^{\prime} 17^{\prime \prime}\right)$ \\
\hline
\end{tabular}

To estimate the probability of chance coincidence we can multiply the relative area of the large error box (that is $\Omega / 4 \pi \mathrm{sr}=17.1 \mathrm{sq}$. deg/ $41252 \mathrm{sq}$. deg $=4.1 \times 10^{-4}$ ) by the probability to have two bursts in $t \lesssim 1500 \mathrm{~s}$ (that is, for the 2008 IPN detection rate, R, of $\sim 250$ GRBs/year, $p=1-\exp (-R t) \simeq 0.012)$ to get $\sim 5 \times 10^{-6}$. Multiplying this number by the total number of GRBs detected by the 3rd IPN so far $(\sim 6000)$, we get a 3\% probability of observing such a close (in time and on the sky) pair of IPN events for the entire 3rd IPN history. Actually this probability is significantly overestimated since we counted all IPN bursts, rather than just those bright enough to be detected by the least sensitive instrument in the IPN, MO HEND.

This simple estimate shows that these episodes likely belong to the same ultra-long GRB.

\section{Spectral analysis and energetics}

The Konus-Wind spectral data from 20 to $1200 \mathrm{keV}$ for the first episode were fitted by the

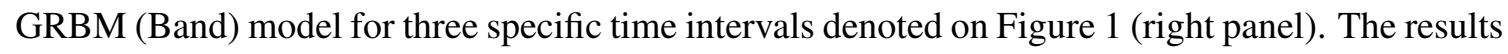
are given in Table $\square$. All spectra have a high-energy power-law tail with $\beta \sim-2.3$. The spectrum of the weaker pulses at $\sim \mathrm{T}_{0}+117 \mathrm{~s}$ is significantly softer (in terms of $E_{\text {peak }}$ ) than the spectrum of the initial bright pulse.

Using the parameters of the time-integrated spectrum (Int. C), the calculated fluence of this episode is $(1.43 \pm 0.04) \times 10^{-4} \mathrm{erg} \mathrm{cm}^{-2}$, and the peak flux is $(7.76 \pm 0.38) \times 10^{-6} \mathrm{erg} \mathrm{cm}^{-2} \mathrm{~s}^{-1}$ as measured from $10.4 \mathrm{~s}$ on a $1 \mathrm{~s}$ timescale (both in the $20-1000 \mathrm{keV})^{2}$.

There are no spectral data for the second episode. Comparison of the counts accumulated over several time intervals by different instruments shows that the hardness of the second episode is

\footnotetext{
${ }^{2}$ in the $20 \mathrm{keV}-10 \mathrm{MeV}$ band they are $(2.01 \pm 0.27) \times 10^{-4} \mathrm{erg} \mathrm{cm}^{-2}$ and $(1.08 \pm 0.10) \times 10^{-5} \mathrm{erg} \mathrm{cm}^{-2} \mathrm{~s}^{-1}$.
} 
Table 2: Spectral parameters of GRB 080407 for three time intervals denoted on Fig. 1 (right panel). All errors are at $90 \% \mathrm{CL}$.

\begin{tabular}{crrlclc}
\hline Int & Tstart (s) & $\mathrm{dT}(\mathrm{s})$ & $\alpha$ & $E_{\text {peak }}(\mathrm{keV})$ & $\beta$ & $\chi^{2} /$ dof (null hypothesis probability) \\
\hline $\mathrm{A}$ & 0 & 43 & $-1.02 \pm 0.06$ & $325_{-25}^{+29}$ & $-2.43_{-0.27}^{+0.16}$ & $60.8 / 59(0.41)$ \\
$\mathrm{B}$ & 117 & 41 & $-1.49_{-0.37}^{+0.76}$ & $114_{-44}^{+77}$ & $-2.25(<-2.02)$ & $48.3 / 59(0.84)$ \\
$\mathrm{C}$ (total) & 0 & 158 & $-1.15_{-0.09}^{+0.10}$ & $287_{-35}^{+42}$ & $-2.35_{-0.40}^{+0.20}$ & $57.1 / 59(0.54)$ \\
\hline
\end{tabular}

Table 3: Ultra-long GRBs

\begin{tabular}{|c|c|c|c|c|c|c|c|}
\hline GRB & $\begin{array}{l}\text { Tstart } \\
\text { s UT }\end{array}$ & $\begin{array}{c}\mathrm{dT} \\
\mathrm{s}\end{array}$ & $\begin{array}{l}\text { Fluence } \\
\mathrm{erg} \mathrm{cm}^{-2}\end{array}$ & lc shape & $\mathrm{z}$ & $\begin{array}{l}E_{\text {iso }} \\
\text { erg }\end{array}$ & Refs. \\
\hline 840304 & $?$ & $\sim 1200$ & $\sim 2.8 \times 10^{-3}$ & $\begin{array}{l}\text { Two broad pulses }(\sim 200 \mathrm{~s}) \\
+ \text { extended tail }(\sim 1000 \mathrm{~s})\end{array}$ & - & $\begin{array}{l}\sim 7.6 \times 10^{54} \\
(\text { for } \mathrm{z}=1 \text { ) }\end{array}$ & [䧃] \\
\hline 971208 & $\begin{array}{l}28092 \\
(07: 48: 12)\end{array}$ & $\sim 2500$ & $(2.55 \pm 0.11) \times 10^{-4}$ & $\begin{array}{l}\text { Single smooth FRED-like } \\
\text { pulse }\end{array}$ & - & $\begin{array}{l}\sim 6.9 \times 10^{53} \\
\text { (for } \mathrm{z}=1 \text { ) }\end{array}$ & [四, 回, 四] \\
\hline 020410 & $\begin{array}{l}38380 \\
(10: 39: 40)\end{array}$ & $\sim 1600$ & $\sim 2.8 \times 10^{-5}$ & Multi-episode & $\simeq 0.5$ & $\sim 1.8 \times 10^{52}$ & [Q, 口] \\
\hline 060814B & $\begin{array}{l}37070 \\
(10: 17: 50)\end{array}$ & $\sim 2700$ & $(2.35 \pm 0.22) \times 10^{-4}$ & $\begin{array}{l}\text { Single smooth FRED-like } \\
\text { pulse }\end{array}$ & - & $\begin{array}{l}\sim 6.4 \times 10^{53} \\
(\text { for } \mathrm{z}=1 \text { ) }\end{array}$ & [四] \\
\hline 080407 & $\begin{array}{l}74529 \\
(20: 42: 09)\end{array}$ & $\sim 2100$ & $\sim 4.5 \times 10^{-4}$ & Multi-episode & - & $\begin{array}{l}\sim 1.2 \times 10^{54} \\
(\text { for } \mathrm{z}=1)\end{array}$ & this work \\
\hline 091024 & $\begin{array}{l}32161 \\
(08: 56: 01)\end{array}$ & $\sim 1200$ & $(1.13 \pm 0.12) \times 10^{-4}$ & Multi-episode & 1.09 & $\sim 3.5 \times 10^{53}$ & [8, 四，回] \\
\hline
\end{tabular}

closer to the hardness of the weaker pulses of the first episode. Using a conversion factor of 1 SPIACS count $=(4.1 \pm 0.5) \times 10^{-10} \mathrm{erg} \mathrm{cm}^{-2}$ (obtained for the weaker pulses of the first episode; for the initial pulse this factor is $\left.(2.31 \pm 0.04) \times 10^{-10} \mathrm{erg} \mathrm{cm}^{-2} \mathrm{cnt}^{-1}\right)$, the estimated fluence of this episode is $\sim 3 \times 10^{-4} \mathrm{erg} \mathrm{cm}^{-2}$. So the total fluence of GRB 080704 is about $4.4 \times 10^{-4} \mathrm{erg} \mathrm{cm}^{-2}$.

\section{Comparison with other ultra-long GRBs}

Table B] contains a comparison of the parameters of the six classical gamma-ray bursts with durations $>1000$ s. It does not include very long underluminous XRFs like XRF 060218, which are thought to be a different phenomenon from classical GRBs. The table also does not list several ultra-long GRB candidates found in the BATSE data [ए]], since it is difficult to establish reliably that the various emission episodes belong to the same GRB.

Only a short note on GRB 840304 has been published, so it is not quite clear whether it really belongs to the class of ultra-long GRBs and has a very large fluence.

In common these ultra-long GRBs have large fluences (except GRB 020410 which has rather moderate fluence) due to their very long durations and rather hard spectra with $E_{\text {peak }}$ of hundreds of $\mathrm{keV}$. But they have quite different morphologies: GRB 971208 and GRB 060814B shows a single, smooth, FRED-like pulse, whereas GRB 020410, GRB 080407, and GRB 091024 show several emission episodes separated by long period(s) of quiescence.

There is also a difference in the light curves of GRB 080407 and two other multi-episode bursts: GRB 080407 shows a bright initial pulse followed by substantially weaker pulses, whereas GRB 020410 and GRB 091024 do not display a decrease of pulse intensities with time (on the contrary, the last pulse of GRB 091024 is brighter that the first one). But in all three cases the initial pulse is significantly harder than subsequent ones. 


\section{Summary}

Only a few ultra-long GRBs (with durations $>1000$ s) have been reported to date. We have presented observations of GRB 080407, probably the longest multi-episode GRB detected so far. The measured burst fluence of $\sim 4 \times 10^{-4} \mathrm{erg} \mathrm{cm}^{-2}(20-1000 \mathrm{keV})$ is among the largest observed in long GRBs. This burst demonstrates similarities with other ultra-long bursts: a long quiescent time between the episodes $(\sim 1500 \mathrm{~s})$, spectral evolution from hard initial pulse to significantly softer subsequent pulses, and a large fluence. The duration of the second emission episode of GRB 080704 (following the quiescent time) is substantially longer that the duration of the first episode, which is a common feature of long GRBs. The existence of such long quiescent times may favor the dormant inner engine scenario over wind modulation models []].

Without knowledge of the burst redshift it is not possible to determine the rest frame properties of the burst, but its large fluence and high $E_{\text {peak }}$ suggest a moderate $\mathrm{z}$ of $\sim 1-2$ (for $\mathrm{z}=1 E_{\text {iso }} \sim 1.2 \times$ $10^{54} \mathrm{erg}$ ). In such a case, the rest frame burst duration of $\sim 1000 \mathrm{~s}$ and the rest frame quiescence time of $\sim 500 \mathrm{~s}$ can pose a problem for some central engine models in the framework of the collapsar scenario. Specifically such long durations might be hard to explain in the magnetar model of long GRBs.

The Konus-Wind experiment is supported by a Russian Space Agency contract and RFBR grant 12-02-00032a. KH is grateful for IPN support under the following NASA grants: NNX07AR71G (MESSENGER), NNX08AN23G (Swift), and NNX08AC90G (INTEGRAL).

\section{References}

[1] Connaughton, V., et al., GRB 971208, IAUC 6785 (1997)

[2] Drago, A., \& Pagliara, G., Quiescent Times in Gamma-Ray Bursts: Hints of a Dormant Inner Engine, ApJ, 665 (2007) 1227

[3] Giblin, T. W., et al., Extended Power-Law Decays in BATSE Gamma-Ray Bursts: Signatures of External Shocks?, ApJ, 570 (2002) 573

[4] Golenetskii, S., et al., Konus-Wind and Konus-RF observations of GRB 091024, GCN Circ., 10083 (2009)

[5] Gruber, D., et al., Fermi/GBM observations of the ultra-long GRB 091024. A burst with an optical flash, $A \& A, \mathbf{5 2 8}$ (2011) A15

[6] Klebesadel, R. W., Laros, J. G., \& Fenimore, E. E. The Unusual Gamma-Ray Burst of March 4, 1984, $B A A S, 16$ (1984) 1016

[7] Levan, A., et al., GRB 020410: A Gamma-Ray Burst Afterglow Discovered by Its Supernova Light, ApJ, 624 (2005) 880

[8] Marshall, F. E., et al., GRB 091024: Swift detection of a burst, GCN Circ., 10062 (2009)

[9] Nicastro, L., et al., Multiwavelength study of the very long GRB 020410, A\&A, 427 (2004) 445

[10] Pal'shin, V. et al., Extremely long hard bursts observed by Konus-Wind, AIP Conference Proceedings, 1000 (2008) 117

[11] Tikhomirova, Ya. Yu., \& Stern, B. E., Superlong Gamma-Ray Bursts, Astronomy Letters, 31 (2005) 291 